\title{
Review of nutrient actions on age-related macular degeneration
}

\author{
Stefania Zampatti ${ }^{a}$, Federico Ricci ${ }^{b}$, Andrea Cusumano ${ }^{b}$, Luigi Tonino Marsella ${ }^{a}$, \\ Giuseppe Novelli ${ }^{a, c}$, Emiliano Giardina ${ }^{a, d, *}$ \\ a Department of Biomedicine and Prevention, School of Medicine, University of Rome "Tor Vergata", Rome, Italy \\ b UOSD Patologia retinica Fondazione PTV "Policlinico Tor Vergata", Rome, Italy \\ c S. Pietro Fatebenefratelli Hospital, Rome, Italy \\ d Laboratorio di Genetica Molecolare UILDM, Fondazione Santa Lucia, Rome, Italy
}

\section{A R T I C L E I N F O}

\section{Article history:}

Received 21 May 2013

Revised 11 October 2013

Accepted 28 October 2013

Keywords:

Age-related macular degeneration

Disease susceptibility

Antioxidants

Nutrients

Nutrition surveys

Food habits

\begin{abstract}
A B S T R A C T
The actions of nutrients and related compounds on age-related macular degeneration (AMD) are explained in this review. The findings from 80 studies published since 2003 on the association between diet and supplements in AMD were reviewed. Antioxidants and other nutrients with an effect on AMD susceptibility include carotenoids (lutein and zeaxanthin, $\beta$-carotene), vitamins (vitamin A, E, C, D, B), mineral supplements (zinc, copper, selenium), dietary fatty acids [monounsaturated fatty acids, polyunsaturated fatty acids (PUFA both omega-3 PUFA and omega-6 PUFA), saturated fatty acids and cholesterol], and dietary carbohydrates. The literature revealed that many of these antioxidants and nutrients exert a protective role by functioning synergistically. Specifically, the use of dietary supplements with targeted actions can provide minimal benefits on the onset or progression of AMD; however, this does not appear to be particularly beneficial in healthy people. Furthermore, some supplements or nutrients have demonstrated discordant effects on AMD in some studies. Since intake of dietary supplements, as well as exposure to damaging environmental factors, is largely dependent on population habits (including dietary practices) and geographical localization, an overall healthy diet appears to be the best strategy in reducing the risk of developing AMD. As of now, the precise mechanism of action of certain nutrients in AMD prevention remains unclear. Thus, future studies are required to examine the effects that nutrients have on AMD and to determine which factors are most strongly correlated with reducing the risk of AMD or preventing its progression.
\end{abstract}

(c) 2014 Elsevier Inc. All rights reserved.

\section{Introduction}

Age-related macular degeneration (AMD) is one of the leading causes of progressive, bilateral blindness in elderly individuals.
AMD can be classified as non-exudative (dry) or exudative (wet). The pathogenesis of dry AMD involves the presence of extracellular debris (drusen) over the retinal pigmented epithelium (RPE). Upon ophthalmoscopic examination, the drusen

\footnotetext{
Abbreviations: AMD, age-related macular degeneration; DHA, docosahexaenoic acid; DRI, dietary reference intake; EPA, eicosapentaenoic acid; HDL, high-density lipoproteins; LDL, low-density lipoproteins; MPOD, macular pigment optical density; MUFA, monounsaturated fatty acid; OR, odds ratio; ROS, reactive oxygen species; RPE, retinal pigmented epithelium; RR, relative risk; SFA, saturated fatty acid.

* Corresponding author. Tel.: +39 067259 6030; fax: +39 0620427313.

E-mail address: emiliano.giardina@uniroma2.it (E. Giardina).
} 
appear as yellowish lesions in the macula. The classification of drusen is based on both dimensional and morphological criteria; drusen may be small ( $<63 \mu \mathrm{m}$ in diameter), medium (63-124 $\mu \mathrm{m})$, or large $(>124 \mu \mathrm{m})$ and can have a hard or soft appearance along the margins [1,2].

The progression of drusen characteristically involves an increase and confluence of more drusen in a single lesion. The diffusion and growth of drusen determine the detachment of RPE with a loss of photoreceptors in the central macula and subsequent blindness. Dysfunction of the RPE, Bruch's membrane, and the choroid are the principal mechanisms involved in the pathogenesis of AMD (Fig. 1) [3]. Environmental and genetic factors influence the events underlying the disease and modify the individual risk factors of developing AMD. Due to the interaction between genetic and environmental factors, the etiology of AMD is multifactorial [4]. As with other complex diseases, recent genome-wide studies have established and confirmed many genetic variants associated with a higher risk for AMD [5-9]. In this review, we summarize the effects that dietary practices and nutritional supplements have on the susceptibility of AMD.

\section{Summary of the literature cited}

This review included electronic searches of the PubMed, Medline, Scopus, and Google scholar databases, using the following search terms: "AMD," "supplements," "antioxidants," "carotenoids," "vitamins," "diet," "intake," "susceptibility," and "risk" in various combinations. These electronic searches were limited to literature within the last 10 years; however, the biological mechanism of nutrients constitutes an exception since the time-limit was removed. This search was supplemented by manually searching the reference lists of original studies and reviews. Herein, we present a summary of the literature on the relationship between nutritional



Fig. 1 - Development of AMD disease. AMD is mainly due to photochemical damage and oxidative stress. In the figure it is represented by the cellular assessment of the normal macula (left side), the dry AMD (center), and the wet AMD (right side). In the left side of the image, the cells in the macula are normally represented. In the dry form of AMD (center), the drusen (composed of a core of glycolipids and glycoconjugates) impairs the metabolic connection between the choroid and the upper layers of macula, leading to degeneration of RPE and photoreceptors. In the wet form of AMD (right side), the production of neovascular factors determines the formation of choroidal neovascularization (CNV) with subsequent fluid leakage and major degeneration of RPE and photoreceptors. In the normal ageing process, the lipids accumulate in the Bruch's membrane, causing a thickening of the membrane and improving the oxidative distress. Moreover, the Bruch's membrane is lacking of an adequate intrinsic antioxidant system. Furthermore, the lipids can bind the macrophages inducing the secretion of the vascular endothelial growth factor (VEGF). The production of inflammation factors improves the damage to the RPE and photoreceptors and induces the formation of functional microvascular networks (choroidal neovacularization). Antioxidant factors might prevent the disease and delay its progression. In the upper portion of the image, the pool of nutrients involved in the onset (left side) and progression (right side) of the AMD are listed. 
factors and AMD. Moreover, the review summarizes the potential roles of nutritional supplements in determining the biological effects of natural products on AMD (Fig. 1). In this review, it is revealed that many supplements have the potential to be protective for AMD and may afford some protection to the dangerous effects of oxidative stress. Antioxidants with an effect on AMD susceptibility include carotenoids (lutein and zeaxanthin, $\beta$-carotene), vitamins (vitamin A, E, C, D, B), mineral supplements (zinc, copper, selenium), dietary fats [monounsaturated fatty acids (MUFA) and polyunsaturated fatty acids (PUFA both omega-3 and omega-6), saturated fatty acids (SFA), cholesterol], and dietary carbohydrates. The literature indicates that many antioxidants exert a protective role by functioning in combination with other nutrients or compounds that have some antioxidant activity. Specifically, the introduction of dietary supplements can provide some benefit on the onset or progression of AMD; however, these may not be particularly valuable in healthy people. In this review, we found that carotenoids, such as lutein and zeaxanthin, were the most investigated. The antioxidant effect of these substances is related to the content in the macular pigment, which protects the photoreceptor cell layer from light damage by filtering blue light. Vitamin supplements show mixed results. Instead of a low risk, high intakes of vitamins $A$ and $C$ have been reported in association with a high risk of AMD. Vitamin E levels are correlated to the intake of other nutrients, and these varied results could be due to the difficulty of singular analysis. Promising results were obtained for vitamins D and B (folic acid-vitamin $B_{9}$, pyridoxine-vitamin $B_{6}$, and cyanocobalaminvitamin $B_{12}$ ). The literature on mineral supplements revealed a slight effect of zinc on the prevention of AMD progression; however, copper and selenium, even when investigated in large studies, failed to demonstrate any influence on AMD susceptibility and progression. Likewise, dietary fats and carbohydrates demonstrated few effects in the prevention of AMD onset and progression. Indeed, the dietary intake of supplements, as well as exposure to damaging environmental factors (such as blue light, UV rays, etc.), are largely dependent on population habits and geographical localization. Nevertheless, a healthy diet appears to be the best strategy to reduce the risk of developing AMD. These findings suggest that physicians should pay close attention when prescribing dietary supplements in the prevention of AMD. Further studies are needed to elucidate the role of individual nutrients and supplements on a patient's risk in the development or progression of AMD. Since one of the main causes of AMD is thought to be related to oxidative stress or an imbalance in pro- and antioxidant factors, we first focus on this condition contributing to AMD.

\section{Oxidative stress in the pathogenesis of AMD and antioxidant nutrients}

Within the last century, several studies have elucidated how light can produce physiological and/or molecular damage to the eye. Oxygen exacerbates this damage via free-radical chain reactions $[10,11]$. This photochemical damage typically involves a compromised region in the macula in patients with
AMD. Thus, the main mechanism inducing damage to the retina affected by AMD is oxidative stress, which is defined as an excess of reactive oxygen species (ROS). ROS production is increased in elderly individuals, and these individuals also demonstrate a reduction in antioxidant defenses. Retinal tissues have several characteristics that predispose an individual to ROS-induced damage. These characteristics include: (i) an abundance of PUFA, (ii) exposure to light (which is a strong oxidizing agent), and (iii) a high metabolism (as the retina requires a large quantity of oxygen).

In the retina, these processes generate free radicals, which are activated forms of oxygen and potentially dangerous due to their role in the oxidative process. Several studies have investigated the protective role of numerous micronutrients and macronutrients in modifying the individual risk factors for developing AMD.

In the search for a specific protective role of antioxidant agents in AMD, the addition of antioxidants to the diet has been the focus of several trial studies. In the human body, several mechanisms have been described in which selected compounds can prevent the formation of free radicals and/or their harmful activity. The intake of these compounds, either through the diet or as a supplement, may modify an individual's susceptibility to AMD.

Several different compounds with antioxidative activities are provided via the diet. The major dietary compounds that possess some aspect of antioxidant activity are carotenoids, vitamin C (ascorbate), and vitamin E (tocopherols) [12]. The effects that other vitamins, minerals (zinc, copper, and selenium), lipids, and carbohydrates have on the overall risk of developing AMD have also been described. In the following sections, we present the nutrients and their actions in AMD and summarize the current knowledge available.

\section{Carotenoids and AMD}

\subsection{Lutein and zeaxanthin}

Upon ophthalmoscopic examination, the macula appears as a yellow spot on the retina. This yellow coloration is due to the presence of carotenoid pigments, such as lutein and zeaxanthin. An increase in the intake of these carotenoids may modify the overall risk of AMD via the protection of retinal tissues from photo-oxidative damage. In fact, the macular pigment contains lutein and zeaxanthin, which protects the photoreceptor cell layer from light damage by filtering blue light $[11,13]$.

Some of the dietary sources of lutein and zeaxanthin have been largely investigated as a protective factor in AMD. Primary studies have focused on the dietary intake of carotenoids, which is obtained from vegetables (eg, kale, spinach, and Brussels sprouts) [11,14], as well as supplements. As expected, however, the dietary contribution of lutein and zeaxanthin is largely dependent on population and dietary habits [15]. The first evidence of an association between the consumption of fruits and vegetables and the risk of AMD was reported in 1988, with the publication of data obtained from the first National Health and Nutrition Examination Survey [16]. 
A case-controlled study focusing on the effect of carotenoids in AMD was performed by the Eye Disease CaseControlled Study Group [17]. Between 1986 and 1990, this study group enrolled 421 patients with neovascular AMD and 615 controls through a multicenter collaboration that involved five medical centers in the United States. The measurement of serum levels of carotenoids revealed that high levels were associated with a reduced risk of neovascular AMD. In particular, the odds ratios for lutein/zeaxanthin, $\beta$-carotene, alpha-carotene, and cryptoxanthin ranged from 0.3 to 0.5 [17]. However, there was no general consistency across outcomes. Some studies failed to identify a correlation between AMD and the intake of vegetables, antioxidant vitamins, and/or carotenoids; and other studies reported a direct association between the higher intake of lutein/zeaxanthin from foods and a reduced likelihood of AMD [13,18,19]. Notably, a large study of 4519 participants, performed by the Age-Related Eye Disease Study Research (AREDS) Group, reported a low likelihood of AMD in people with a high dietary intake of lutein/zeaxanthin [20].

If the effects of carotenoids on the risk of AMD are not fully understood, then their role on disease progression is similarly unclear. In 2007, Robman and colleagues reported that increased intakes of dietary lutein, zeaxanthin, and omega-3 fatty acids were associated with AMD progression [21]. Subsequently, several studies reported the opposite results [22]. In particular, a recent study published by $\mathrm{Ma}$ et al reported that the intake of lutein and zeaxanthin supplements in patients with early AMD could improve the macular pigment optical density (MPOD) and visual function [23]. Moreover, a recent PIMAVOSA (PIgment MAculaire chez le VOlontaire SAin [macular pigment in the healthy volunteer]) study reported a significant correlation between MPOD and the plasma levels of lutein and zeaxanthin $(r=0.35, P<.001$, and $r=0.30, P<.005$, respectively) [24]. Also, in a large metaanalysis published by Ma et al [25], it was revealed that a high dietary intake of lutein and zeaxanthin was useful in reducing the risk of late AMD, with no effect on early AMD.

Furthermore, cigarette smoking, a well-known risk factor for AMD, was associated with a reduction in the serum levels of carotenoids, $[15,26]$ and when combined with a low dietary intake of carotenoids, is a confirmed risk factor for AMD. However, despite this evidence, the interplay between smoking and carotenoid levels on the modification of individual risk has yet to be elucidated.

Conversely, it is well known that there is a direct correlation between low serum carotenoid levels and inflammation and obesity $[27,28]$. Carotenoids are transported by low-density lipoproteins and high-density lipoproteins, suggesting that the lipid profile may influence the transport and delivery of these carotenoids to the retina with a consequential effect on macular pigment [29]. Many authors have considered the MPOD as a marker of eye health, and there is some evidence that carotenoid supplementation might improve this marker [30].

From a genetic perspective, there are no confirmed interactions between known associated gene...tic variants of AMD development and carotenoid levels. Seddon et al found no significant interactions between lutein and the polymorphism rs1410996 in the LIPC gene [31]. Subsequently, Ho et al reported an interaction between the $\mathrm{CFH}$ Y402H genotype and lutein/zeaxanthin, as well as with zinc, $\beta$-carotene, and docosahexaenoic acid (DHA)/eicosapentaenoic acid (EPA). These authors reported a significant interaction between very common genetic variants (CFH Y402H and ARMS2 A69S) and dietary antioxidant intake in AMD [32]. However, no other studies have replicated these data. Further analyses investigating the interaction between nutritional habits and genetic susceptibility in AMD are required prior to the routine application of lutein/zeaxanthin in AMD treatment.

\section{2. $\beta$-Carotene}

$\beta$-Carotene is a carotenoid with vitamin A activity and often referred to as a provitamin A since, after absorption, it is cleaved by specific enzymes and contributes to the supply of vitamin A in humans. Because of its antioxidative properties, $\beta$-carotene has been included in multivitamin supplements, although its role in the prevention of AMD has yet to be confirmed $[12,20,33]$. Subsequently, it is reported that there is an increased risk for the development of AMD in individuals with a high dietary intake of $\beta$-carotene $[34,35]$. Moreover, several adverse effects of supplementation with $\beta$-carotene have been described, in particular an increase in the likelihood of developing lung cancer. Although some studies have reported no association [36], 2 large trials investigated the effect of dietary supplementation on the risk of lung cancer and found a direct relationship. In the CARET (beta-CArotene and Retinol Efficacy Trial) study, the combination of $\beta$ carotene and retinol (vitamin A) increased the risk of lung cancer and cardiovascular events [37]; while in the ATBC (Alpha-Tocopherol Beta-Carotene) trial, this risk was increased by $16 \%$ after 6 years [38]. Taken together, this evidence discourages the use of $\beta$-carotene in the prevention of AMD due to the potential side effects and poor efficacy in reducing the risk of AMD [35].

\section{Vitamins and AMD}

\subsection{Vitamin A}

Vitamin A plays several roles in the human body and is closely related to carotenoids, which are by-products of vitamin A. Data from the National Health and Nutrition Examination Survey, reported by Goldberg et al, showed a negative association between the frequency of fruit and vegetables rich in vitamin A consumed and the prevalence of AMD [16]. However, several subsequent studies and a large metaanalysis failed to confirm this association [20,22,39,40], and unexpectedly, one report even suggested an increased risk in the development of late AMD in participants who took vitamin A [41]. With these conflicting results, more research is needed regarding the association between vitamin A and AMD.

\subsection{Vitamin $E$}

Vitamin E consists of several antioxidants; however, alphatocopherol is the only form largely present in blood and tissue. alpha-Tocopherol is the form of vitamin $\mathrm{E}$ that is preferentially 
absorbed and accumulated and is found in vegetable oils, nuts, whole grains, and green leafy vegetables [42]. Side effects of vitamin E supplementation include a potential antiplatelet anticoagulant effect, which is an antagonizing effect of fatsoluble vitamins, and heart failure in patients with preexisting vascular disease and diabetes. Furthermore, vitamin E might increase the risk of developing prostate cancer $[43,44]$.

Although there was no association found between vitamin E and AMD in the Eye Disease Case-Control Study [17], in the POLA (Pathologies Oculaires Liées à l'Age) study by Delcourt et al, a reduction in the risk of late AMD in individuals with enhanced plasma levels of alpha-tocopherol was demonstrated [39]. Several studies report a weak or non-existent association between alpha-tocopherol levels/vitamin E supplements and the risk of AMD [33,45]. The results from the AREDS report no. 22 demonstrated an association between the high intake of $\beta$-carotene and vitamins $C$ and $E$, as well as a reduced likelihood of neovascular AMD [20]. A meta-analysis published by Chong et al of randomized controlled trials reported an odds ratio of 0.75 , which was obtained by pooling the results from two studies: the Rotterdam eye study [46] and the Beaver Dam eye study [40,47]. The results from subsequent meta-analyses performed by Chong et al [40] and by Evans and Lawrenson [35] did not support a specific role for vitamin $E$ in the prevention of AMD. To explain the discordant data of dietary vitamin E compared with the supplements, Evans and Lawrenson concluded that dietary vitamin $\mathrm{E}$ might be a marker for other nutrients with a role in the susceptibility of AMD [35]. In fact, plasma alphatocopherol levels are related to carotenoids and vitamin C intake [48], supporting the theory by Evans and Lawrenson regarding the varying data.

\subsection{Vitamin C}

Vitamin C (ascorbate) is an effective antioxidant that protects proteins, lipids, carbohydrates, and nucleic acids from free radicals and ROS damage. However, no significant association between vitamin $C$ intake and AMD was observed in the Eye Disease Case-Control Study Group or in the POLA study $[17,39]$. The lack of effect that vitamin $C$ had on primary prevention of AMD was further supported by a Cochrane analysis conducted by Evans and Lawrenson [35]. Nevertheless, SanGiovanni et al reported a reduced likelihood of neovascular AMD in subjects reporting high intakes of $\beta$ carotene and vitamins $C$ and $E$ [20]. In the meta-analysis performed by Chong et al, the pooled odds ratio of vitamin $\mathrm{C}$ supplements was 1.11 (95\% CI, 0.84-1.46), in reference to early AMD [40]. Further research is needed to draw any definite conclusions regarding vitamin $C$ and AMD.

\subsection{Vitamin D}

The specific effect that vitamin D has on AMD was investigated because of the anti-inflammatory properties found in vitamin D. The results from the National Health and Nutrition Examination Survey study showed an association between the plasma levels of vitamin $\mathrm{D}$ and early AMD, with no association reported for advanced AMD [49]. In 2011, Seddon et al reported that a higher dietary intake of vitamin D was associated with less severe AMD and a smaller drusen size, when analyzing monozygotic twins with discordant AMD [50]. More recently, Miller et al reported a protective role of high serum 25-hydroxyvitamin D (25(OH)D) concentrations against early $A M D$, among females participating in the CAREDS (Carotenoids in Age-Related Eye Disease Study) trial [51]. Taken together, these results suggest that further evidence is required to fully dissect the role of vitamin D in AMD.

\subsection{B vitamins}

The B vitamins are a group of vitamins that play important roles in cell metabolism. The dietary intake of folic acid, vitamin $B_{6}$, and vitamin $B_{12}$ can modify homocysteine levels. Hyperhomocysteinemia is a risk factor for several vascular diseases (atherosclerosis, coronary heart disease, venous thromboembolism, and retinal vascular occlusive disease). Kamburoglu et al reported an association between homocysteine levels and AMD. Patients with either dry or exudative AMD presented with high plasma homocysteine. In addition, plasma vitamin $B_{12}$ was lower in patients with exudative $A M D$, compared with controls and patients with dry AMD [52]. In the Blue Mountains Eye study, a comparison between homocysteine, vitamin $\mathrm{B}_{12}$, and folate levels showed an association between serum homocysteine ( $>15 \mu \mathrm{mol} / \mathrm{L}$ ) and an increased likelihood of AMD. Moreover, a similar association was described for low levels of vitamin $\mathrm{B}_{12}$. However, no association was found for folate levels [53]. More recently, a randomized trial of 5442 females revealed a decreased risk of AMD in participants who took daily supplements of folic acid (vitamin $\mathrm{B}_{9}$ ), pyridoxine (vitamin $\mathrm{B}_{6}$ ), and cyanocobalamin (vitamin $B_{12}$ ) [54]. As with the previously discussed vitamin groups, more research is needed prior to drawing any conclusions regarding the efficacy of using $B$ vitamins to prevent or treat AMD.

\section{Mineral supplements and AMD}

\subsection{Zinc}

Zinc is a trace element that is essential for the human body, in which its antioxidative functions act as a cofactor for superoxide dismutase and catalase. Zinc is stored and transferred in metallothioneins. High zinc intake (>50 mg/ day) can induce copper deficiency. In fact, metallothioneins, which bind to zinc, have a strong affinity for copper; where high levels of metallothioneins, induced by zinc excess, may cause a decrease in intestinal copper absorption. Similarly, copper intake can affect the zinc nutritional status [42], and zinc supplementation might even affect cysteine levels $[55,56]$.

The first evidence of the role of zinc supplementation in AMD was published in 1988. In this prospective trial of 151 patients, it showed that the likelihood of vision loss was lower in the zinc-treated group when compared with the placebo group, after a follow-up evaluation of 12 to 24 months [57]. Subsequently, the results of several studies disputed the role of zinc in AMD susceptibility [58]. However, results from the Beaver Dam Eye Study confirmed a weak protective effect of zinc on some forms of AMD [45], with no overall association 
with early AMD [47]. Subsequently, Klein et al reported a weak association between late AMD and the incidental use of vitamins $\mathrm{A}, \mathrm{C}$, and $\mathrm{E}$ and zinc [41].

Furthermore, a prospective study performed by Cho et al failed to identify an association between zinc intake (food or supplements) and AMD risk [59]. Interestingly, different results were provided by the Blue Mountains Eye Study. In fact, in one of the initial reports, Flood et al described a lack of an association between dietary zinc and the risk of early AMD [60]; similarly no association was reported for zinc supplements [61]. Subsequently, after a long-term (up to 10 years) follow-up evaluation of participants in the Blue Mountains Eye Study, Tan et al described a protective effect of zinc against AMD (any AMD, relative risk [RR] 0.56; early AMD, RR 0.54) [34].

In 2005, van Leeuwen reported that the combined dietary intake of beta carotene, vitamins $\mathrm{C}$ and $\mathrm{E}$, and zinc was associated with a reduced risk of AMD [46]. The effect and tolerance of zinc supplementation was also investigated $[62,63]$, and it appeared to improve macular function in patients with dry AMD [63]. The beneficial effect of zinc supplements was also reported in a recent analysis conducted by Beatty et al in a trial that focused on carotenoids with coantioxidants in age-related maculopathy. Patients who took supplementation with lutein, zeaxanthin, vitamin C, vitamin E, copper, and zinc demonstrated a slower progression and enhanced visual acuity compared with the placebo group [64]. In summary, taking into consideration the studies cited above even with the varying results regarding the benefit of zinc on AMD, many studies appear to support the value of zinc supplementation overall.

\subsection{Copper}

Copper is an essential trace element with the specific ability to easily accept and donate electrons; thus, it plays an important role in oxido-reduction and the scavenging of free radicals [42]. Copper was added to the AREDS formulation due to its strong link with zinc levels; high levels of zinc intake may cause copper deficiency anemia [13]. The involvement of copper in AMD was first proposed by Newsome but was never definitively confirmed $[57,65]$. Thus, further studies are required to determine the putative effect of copper on the pathogenesis, susceptibility, and progression of AMD.

\subsection{Selenium}

Selenium is a trace element that is found in several enzymes in humans and has a well-recognized antioxidative function [66]. In the past years, several studies have focused on the effect of selenium in AMD; however, these results have not been convincing $[17,67]$. Therefore, due to the lack of recent large studies, the role of selenium and its enzymes in AMD susceptibility remains unclear [35].

\section{Antioxidant combinations and AMD}

The AREDS trial introduced treatment with oral supplements to prevent the onset or progression of AMD. Supplements in the AREDS formula include vitamins A, C, and E, and zinc, for their antioxidative effects. The dosage of AREDS supplements was higher than the recommended Dietary Reference Intake. Although AREDS and AREDS-like supplements appear to protect against AMD, several potential risks of oral supplementation have been described (eg, vitamin $\mathrm{C}$ and kidney stones; vitamin $\mathrm{E}$ and fatigue, muscle weakness, decreased thyroid function, and increased hemorrhagic stroke risk; $\beta$ carotene and increased lung cancer risk in smokers and yellow discoloration of skin; and zinc with anemia, decreased serum high-density lipoprotein cholesterol, and stomach upset) [68]. The results from the Rotterdam Study have provided evidence that a high dietary intake of select antioxidant nutrients substantially reduces the risk of AMD [46]. However, a recent review published by Evans and Lawrenson in the Cochrane Collaboration provided evidence that such nutrients had no effect in reducing the risk of AMD.

\section{Dietary fats and carbohydrates in AMD}

Several studies have attempted to elucidate the effect of different dietary habits on susceptibility to AMD. In particular, several efforts have been made in defining the relationship between dietary fat intake and the risk of AMD. Fats consumed from the diet can be classified as either unsaturated fatty acids or saturated fatty acids.

\subsection{Omega-3 and omega-6 PUFA}

One of the first fats investigated in AMD were the long-chain omega-3 PUFA ( $n-3$ PUFA) due to their involvement in coronary heart disease and other types of degenerative retinal diseases [69]. The n-3 PUFA primarily investigated in AMD includes alpha-linolenic acid, DHA, and EPA. The omega-6 PUFA (n-6 PUFA) which includes linoleic acid and arachidonic acid.

In a large prospective study published by Cho et al, a positive association between dietary fat intake and AMD was reported. The study included 42,743 females and 29,746 males who were followed for 10 to 12 years; 567 of the subjects developed AMD. The amount of dietary fat intake was assessed using a food-frequency questionnaire. Statistical analyses revealed an increased risk of AMD in subjects with a high dietary fat intake. Foods that mainly contributed to the risk of AMD were beef, pork, and lamb; in particular, total fat and $\alpha$-linolenic acid were risk factors. In contrast, DHA intake showed a modest inverse correlation with AMD risk, confirming the previously reported beneficial actions of fish intake [70]. In the same year, conflicting results were reported. Heuberger et al found no association between dietary fat intake and AMD [71], while Seddon et al confirmed the role of several factors as risk factors [72] by evaluating the effect of the total and subtypes of fat on AMD. Moreover, Seddon et al confirmed the protective role of n-3 PUFA, as well as the enhanced risk of AMD, in individuals with high levels of MUFA and PUFA. Interestingly, the beneficial effect of omega-3 fatty acids and fish intake was diminished in subjects with high levels of linoleic acid intake (an omega-6 fatty acid) [73].

Although some studies failed to confirm the association between fat intake and AMD [71], other studies have found a significant association between dietary fat intake and disease 
[33,73-75]. In particular, the AREDS study confirmed the protective effect of dietary total n-3 PUFA intake in neovascular AMD [73] and central geographic atrophy [75]. Furthermore, Sangiovanni et al confirmed the inverse association between dietary DHA intake and fish consumption and the risk of neovascular disease. Conversely, dietary arachidonic acid intake was directly associated with neovascular AMD prevalence [73]. The results from the Blue Mountain Eye Study confirmed these associations, providing further evidence of the protective role of fish, n-3 PUFA, and a low intake of foods rich in linoleic acid [34]. Taken together, these supporting data motivated further studies to investigate the effects of specific fat supplements on AMD. Johnson et al reported a positive relationship between DHA supplementation and an increase in MPOD, after 4 months of treatment [30].

Subsequently, Parekh et al reported results from the Carotenoids in Age-Related Eye Disease Study (CAREDS) [76]. N-6 PUFA intake was reported as being directly associated with intermediate AMD signs. Parekh and colleagues found no association between n-3 PUFA and AMD. Moreover, in the sample analyzed by Parekh et al, a high correlation between the intake of n-3 PUFA and n-6 PUFA was found $(r=0.8)$. Similarly, a PIMAVOSA study reported an association between high plasma levels of total n-3 PUFA and high MPOD $(r=0.22$, $P<.05)$; however, the mechanisms through which n-3 PUFA correlate with MPOD remain to be elucidated. Nevertheless, several hypotheses have been made, such as; the PUFA may modulate the gastrointestinal uptake of lutein and zeaxanthin, their transport by lipoproteins, or their concentration in the macular area [24].

Prospective studies have also demonstrated that high intake of total fat can be a risk factor for progression to advanced AMD (ie, geographic atrophy or neovascular disease) among patients with early or intermediate disease. Similarly, higher fish intake was associated with a lower risk of AMD progression among patients with lower linoleic acid intake [77]. A subsequent study performed on the AREDS population showed a protective effect of DHA and EPA in the progression to advanced AMD. Moreover, consuming a diet rich in DHA protects against the progression of early AMD, particularly among subjects not taking AREDS supplements [78].

Several reviews have attempted to elucidate the role of $n-3$ PUFA in the susceptibility and progression of AMD. However, no significant evidence was provided in these studies [79-81]. A recent review of randomized controlled trials concluded that there was no sufficient evidence to support the role of increasing levels of dietary n-3 PUFA to prevent or slow the progression of AMD [82]. Given these results, both n-3 PUFA and n- 6 PUFA need further studies to determine the impact that these PUFA have on AMD.

\subsection{The MUFA}

High dietary intake of SFA, MUFA, and trans unsaturated fatty acids have been reported to be associated with a marginal increase in the risk of AMD $[69,72,77]$. Previous studies have reported inverse association between MUFA and AMD risk. In particular, Smith et al reported that a high dietary intake of MUFA was a risk factor for early AMD [70], and Parekh et al demonstrated that it was a protective factor for intermediate
AMD [76]. A potential explanation of these contrasting results was reported by the AREDS study group. Report no. 20 demonstrated that the potential of neovascular AMD was increased among individuals with the highest intake of MUFAs, but when modeled as a primary independent variable and other nutrients (DHA, arachidonic acid, and lutein/ zeaxanthin) adjusted for, this association was lost. Similarly, no significant association was found for SFA or cholesterol after adjustment for other nutrients [73].

Nevertheless, it appears that a higher intake of MUFA is associated with the progression of AMD. Similarly, the association between PUFA and progression of the disease was similar but not statistically significant [77].

\subsection{The SFA}

The first evidence of a relationship between SFA and AMD was provided by Mares-Perlman et al, who found an increased risk of early AMD in individuals with the highest intake of SFA and cholesterol [83]. Subsequently, Smith et al reported only a borderline significant association between SFA intake and the risk for early AMD [70]. Similarly, Cho et al described a modest increase in the risk of AMD for subjects with a high dietary intake of saturated fats [69]. However, this association was not confirmed in the study reported by Seddon et al in the same year [72]. Nevertheless, in the AREDS study, Sangiovanni et al described an increased odds ratio of neovascular AMD among subjects with the highest intake of saturated fatty acid [73].

Interestingly, results from the CAREDS trial revealed a correlation between the effects of fat intake and patient age. In fact, in this study, a high intake of saturated fatty acid was found to be associated with an increased prevalence of AMD in females younger than 75 years, while an inverse association was observed in older females [76]. Furthermore, Seddon et al described an association between a higher intake of SFA and the progression of AMD [77].

\subsection{Cholesterol}

Smith et al [70] reported a borderline significant increase in the risk for late AMD in association with high levels of dietary cholesterol intake [70], thus confirming results reported by Mares-Perlman et al [83]. Similarly, the AREDS study described an increased likelihood of developing AMD with an increased cholesterol intake [73]. The results for cholesterol, as well as other lipids, have not yet been confirmed [69].

\subsection{Dietary carbohydrates}

Several studies have investigated the role of carbohydrates in AMD risk by calculating the glycemic index. The glycemic index is a measure of the ability of $50 \mathrm{~g}$ of a specific food to increase blood glucose levels compared to the ability of $50 \mathrm{~g}$ of standard food to increase blood glucose levels [22,84].

Independent data support an effective role of a low dietary glycemic index in preventing the progression of AMD, particularly in people at risk for advanced AMD [78,85-87]. Chiu et al described a synergistic effect of AREDS supplements with a low dietary glycemic index. In particular, individuals at risk for advanced AMD should consume a low glycemic index 
diet, while those also taking AREDS supplements would benefit even more [78]. Similarly, a significant synergistic interaction was described with regard to omega-3 fatty acids and dietary glycemic index; higher intake of omega-3 fatty acids (DHA and EPA) and low dietary glycemic index were associated with an even lower risk for advanced AMD compared to either of the two dietary factors alone [78]. In addition, recent studies on murine models also support these results $[22,88]$.

\section{AMD gaps in knowledge and future research}

\subsection{Gaps in knowledge}

Literature analysis revealed that many of the products exert a protective role against AMD by acting in combination. Several studies have reported the strong link between serum levels of nutrients (i.e., alpha-tocopherol levels with carotenoids and vitamin C). Additionally, the dietary intake of some nutrients is mutually related due to their simultaneous presence in food. Furthermore, the dietary intake of supplements, as well the exposure to environmental damaging factors, is largely dependent on population habits and geographical localization. Case-controlled studies need to optimize samples by equalizing environmental factors (such as food intake, lifestyle, and exposure to external factors). The main weakness of studies on nutritional supplements in AMD is the impossibility to adequately standardize the sample. Many studies collected data on dietary habits using interviews, which are affected by the individual's ability to measure the food. Moreover, food typically contains more than one nutrient and so the dosage of single factors may be very difficult to determine. To determine the effect of single nutrients on the onset and progression of AMD, it is essential to analyze these different studies as a whole and minimize the differences between the samples. By analyzing the literature, it is evident that, although some dietary supplements can provide minimal effects on AMD, they do not appear to be particularly useful in healthy people.

Further efforts have to be made to standardize the samples for dietary habits, lifestyle, and environmental exposures (such as blue light, UV ray, etc). Adequate standardization of the samples might elucidate the slight effect that nutrients have on the development and progression of AMD.

For further comprehension of AMD, it will be useful to investigate the biological effect of nutrients on the pathogenesis of AMD and how genetic variants can condition the response to these effects. At the present, a small number of studies have been performed using dietary and genetic examination, but with poor results. Further analyses are necessary to better understand the pathological mechanisms that underlie AMD pathogenesis and the effect of nutrients on AMD.

\subsection{Future research needs}

The diagnosis and treatment of AMD is predominantly determined by the personal experience of the ophthalmologist. However, current treatments are potentially dangerous and involve oncology and are not free of side effects. Moreover, treatments that are safe (such as nutritional supplements) appear ineffective. Since the most optimal treatment for AMD patients still remains distant from clinical practices, further investigations are required to improve the existing knowledge. Although many studies have investigated the beneficial role of micronutrients in the diet, as well as multivitamin supplements, current knowledge does not support the clinical use of these supplements in patients with AMD. On the basis of this review, it is evident that additional studies are necessary to better elucidate the specific role of nutrients on the pathological onset and progression. We expect that the combined analysis of the nutritional effect of food/supplements and the genetic profile will increase our knowledge about, and thus ability to analyze, the entire system. Many differences in the effect of nutrients could be explained by analyzing the differences in the biological systems involved. In particular, genetic variants can provide minimal modification on the cellular pathway. Thus, taken together, the genetic variants can create a different target for the nutrients. Future studies should take into account these aspects, combining nutrient analyses and genetic profiles in order to determine the different responses provided from varying genetic backgrounds. In this perspective, the stratification of patients based on their genetic profiles could provide relevant explanations of the heterogeneity of the effects shown by the majority of the studies. The standardization of samples for dietary habits, lifestyle, environmental exposures, and genetic profiles can help to define the role of nutrients. The adequate standardization of samples might elucidate the slight effect of nutrients on the development and progression of AMD.

As stated previously, since few studies have been performed that combine examinations on diet and genetic profiles, more research is needed in this area. Knowledge on the molecular pathways of nutrients, according to the genetic identity of the patients, will enable the application of the most optimal treatment for each patient with AMD. Genetic profiling would likely contribute to our understanding of AMD and potentially provide more effective management of the disease, such as the case in HIV $[89,90]$ and HCV $[91,92]$ infection.

\section{Conclusions}

AMD is one of the leading causes of blindness in developed countries. The progression of AMD has social and economic implications. Due to the burden of this disease, efforts have been made to identify strategies that can delay the onset or progression of AMD. Many studies have investigated the beneficial role of micronutrients in the diet as well as multivitamin supplements. However, current knowledge does not support the clinical use of supplements in patients with AMD. Several reviews have analyzed data obtained from case-controlled studies and randomized trials and have also found no evidence of supplements benefiting healthy people $[22,35]$. Evans and Lawrenson emphasized the clinical practice implications by stating: "there is no evidence from randomized controlled trials that healthy people should take antioxidant 
vitamin and mineral supplements to prevent or delay the onset of AMD." [35].

At present, dietary supplements seem to provide minimal effects on the onset or progression of AMD, and safety data are insufficient for the application to the overall population. Similarly, macronutrients in the diet can minimally affect the onset and progression of AMD. Thus, further studies are necessary to better elucidate the specific role of micro- and macronutrients and to identify a common strategy to prevent the onset and progression of AMD. In this perspective, the stratification of patients based on their genetic profiles could provide relevant explanations regarding the heterogeneity of the effects observed in the majority of studies. Currently, it appears that a healthy and balanced diet is the gold standard for all people [93]. Application of these dietary and lifestyle improvements has shown major relevance in individuals with a high risk of AMD, either with or without a family history or genetic profile. Until further definitive research regarding the efficacy of nutrient supplementation and AMD is provided smoking cessation, weight reduction, and a healthy diet appear to be the best strategies to reduce the risk of developing AMD.

\section{Acknowledgment}

This work was supported by the "Macula \& Genoma Foundation-onlus."

\section{R E F E R E N C E S}

[1] Bird AC, Bressler NM, Bressler SB, Chisholm IH, Coscas G, Davis MD, et al. An international classification and grading system for age-related maculopathy and age-related macular degeneration. The International ARM Epidemiological Study Group. Surv Ophthalmol 1995;39:367-74.

[2] Jager RD, Mieler WF, Miller JW. Age-related macular degeneration. N Engl J Med 2008;358:2606-17.

[3] Roth F, Bindewald A, Holz FG. Keypathophysiologic pathways in age-related macular disease. Graefes Arch Clin Exp Ophthalmol 2004;242:710-6.

[4] Giardina E, Sinibaldi C, Novelli G. The psoriasis genetics as a model of complex disease. Curr Drug Targets Inflamm Allergy 2004;3:129-36.

[5] Ricci F, Zampatti S, D'Abbruzzi F, Missiroli F, Martone C, Lepre $\mathrm{T}$, et al. Typing of ARMS2 and CFH in age-related macular degeneration: case-control study and assessment of frequency in the Italian population. Arch Ophthalmol 2009;127:1368-72.

[6] Lepre T, Cascella R, Missiroli F, De Felici C, Taglia F, Zampatti $\mathrm{S}$, et al. Polymorphisms in ARMS2 (LOC387715) and LOXL1 genes in the Japanese with age-related macular degeneration. Am J Ophthalmol 2011;152:326.

[7] Ricci F, Staurenghi G, Lepre T, Missiroli F, Zampatti S, Cascella $\mathrm{R}$, et al. Haplotypes in IL-8 gene are associated to age-related macular degeneration: a case-control study. PLoS One 2013;8: e66978. http://dx.doi.org/10.1371/journal.pone.0066978.

[8] Chen W, Stambolian D, Edwards AO, Branham KE, Othman M, Jakobsdottir J, et al. Genetic variants near TIMP3 and high-density lipoprotein-associated loci influence susceptibility to age-related macular de generation. Proc Natl Acad Sci U S A 2010;107:7401-6.

[9] Neale BM, Fagerness J, Reynolds R, Sobrin L, Parker M, Raychaudhuri S, et al. Genome-wide association study of advanced age-related macular degeneration identifies a role of the hepatic lipase gene (LIPC). Proc Natl Acad Sci U S A 2010;107:7395-400.

[10] Young RW. Solar radiation and age-related macular degeneration. Surv Ophthalmol 1988;32:252-69.

[11] Chalam KV, Khetpal V, Rusovici R, Balaiya S. A review: role of ultraviolet radiation in age-related macular degeneration. Eye Contact Lens 2011;37:225-32.

[12] Stahl W. Macular carotenoids: lutein and zeaxanthin. In: Augustin A, editor. Nutrition and the Eye. , Dev OphthalmolBasel: Karger; 2005. p. 70-88.

[13] Sin HP, Liu DT, Lam DS. Lifestyle modification, nutritional and vitamins supplements for age-related macular degeneration. Acta Ophthalmol 2013;91:6-11.

[14] Sommerburg O, Keunen JE, Bird AC, van Kuijk FJ. Fruits and vegetables that are sources for lutein and zeaxanthin: the macular pigment in human eyes. Br J Ophthalmol 1998;82: 907-10.

[15] Woodside JV, Young IS, Gilchrist SE, Vioque J, Chakravarthy U, de Jong PT, et al. Factors associated with serum/plasma concentrations of vitamins A, C, E and carotenoids in older people throughout Europe: the EUREYE study. Eur J Nutr 2013;52:1493-501.

[16] Goldberg J, Flowerdew G, Smith E, Brody JA, Tso MO. Factors associated with age-related macular degeneration. An analysis of data from the first National Health and Nutrition Examination Survey. Am J Epidemiol 1988;128: 700-10.

[17] Antioxidant status and neovascular age-related macular degeneration. Eye Disease Case-Control Study Group. Arch Ophthalmol 1993;111:104-9.

[18] Cho E, Seddon JM, Rosner B, Willett WC, Hankinson SE. Prospective study of intake of fruits, vegetables, vitamins, and carotenoids and risk of age-related maculopathy. Arch Ophthalmol 2004;122:883-92.

[19] Boeing H, Bechthold A, Bub A, Ellinger S, Haller D, Kroke A, et al. Critical review: vegetables and fruit in the prevention of chronic diseases. Eur J Nutr 2012;51:637-63.

[20] Age-Related Eye Disease Study Research Group, SanGiovanni JP, Chew EY, Clemons TE, Ferris III FL, Gensler G, et al. The relationship of dietary carotenoid and vitamin A, E, and C intake with age-related macular degeneration in a case-control study: AREDS Report No. 22. Arch Ophthalmol 2007;125:1225-32.

[21] Robman L, Vu H, Hodge A, Tikellis G, Dimitrov P, McCarty C, et al. Dietary lutein, zeaxanthin, and fats and the progression of age-related macular degeneration. Can J Ophthalmol 2007;42:720-6.

[22] Weikel KA, Chiu CJ, Taylor A. Nutritional modulation of age-related macular degeneration. Mol Aspects Med 2012;33:318-75.

[23] Ma L, Yan SF, Huang YM, Lu XR, Qian F, Pang HL, et al. Effect of lutein and zeaxanthin on macular pigment and visual function in patients with early age-related macular degeneration. Ophthalmology 2012;119:2290-7.

[24] Delyfer MN, Buaud B, Korobelnik JF, Rougier MB, Schalch W, Etheve $\mathrm{S}$, et al. Association of macular pigment density with plasma $\omega-3$ fatty acids: the PIMAVOSA study. Invest Ophthalmol Vis Sci 2012;53:1204-10.

[25] Ma L, Dou HL, Wu YQ Huang YM, Huang YB, Xu XR, et al. Lutein and zeaxanthin intake and the risk of age-related macular degeneration: a systematic review and meta-analysis. Br J Nutr 2012;107:350-9.

[26] Snodderly DM. Evidence for protection against age-related macular degeneration by carotenoids and antioxidant vitamins. Am J Clin Nutr 1995;62:1448S-61S.

[27] Adams MK, Simpson JA, Aung KZ, Makeyeva GA, Giles GG, English DR, et al. Abdominal obesity and age-related macular degeneration. Am J Epidemiol 2011;173:1246-55. 
[28] Johnson EJ. Obesity, lutein metabolism, and age-related macular degeneration: a web of connections. Nutr Rev 2005;63:9-15.

[29] Loane E, Nolan JM, O'Donovan O, Bhosale P, Bernstein PS, Beatty S. Transport and retinal capture of lutein and zeaxanthin with reference to age-related macular degeneration. Surv Ophthalmol 2008;53:68-81.

[30] Johnson EJ, Chung HY, Caldarella SM, Snodderly DM. The influence of supplemental lutein and docosahexaenoic acid on serum, lipoproteins, and macular pigmentation. Am J Clin Nutr 2008;87:1521-9.

[31] Seddon JM, Reynolds R, Rosner B. Associations of smoking, body mass index, dietary lutein, and the LIPC gene variant rs10468017 with advanced age-related macular degeneration. Mol Vis 2010;16:2412-24.

[32] Ho L, van Leeuwen R, Witteman JC, van Duijn CM, Uitterlinden AG, Hofman A, et al. Reducing the genetic risk of age-related macular degeneration with dietary antioxidants, zinc, and $\omega-3$ fatty acids: the Rotterdam study. Arch Ophthalmol 2011;129:758-66.

[33] Age-Related Eye Disease Study Research Group. A randomized, placebo-controlled, clinical trial of high-dose supplementation with vitamins $\mathrm{C}$ and $\mathrm{E}$, beta carotene, and zinc for age-related macular degeneration and vision loss: AREDS report no. 8. Arch Ophthalmol 2001;119:1417-36.

[34] Tan JS, Wang JJ, Flood V, Rochtchina E, Smith W, Mitchell P. Dietary antioxidants and the long-term incidence of age-related macular degeneration: the Blue Mountains Eye Study. Ophthalmology 2008;115:334-41.

[35] Evans JR, Lawrenson JG. Antioxidant vitamin and mineral supplements for preventing age-related macular degeneration. Cochrane Database Syst Rev 2012;6:CD000253. http: //dx.doi.org/10.1002/14651858 [Available online: http:// onlinelibrary.wiley.com/doi/10.1002/14651858.CD000253.pub3/ full (accessed on 09/01/2013)].

[36] Hennekens CH, Buring JE, Manson JE, Stampfer M, Rosner B, Cook NR, et al. Lack of effect of long-term supplementation with beta carotene on the incidence of malignant neoplasms and cardiovascular disease. N Engl J Med 1996;334:1145-9.

[37] Omenn GS, Goodman GE, Thornquist MD, Balmes J, Cullen MR, Glass A, et al. Effects of a combination of beta carotene and vitamin A on lung cancer and cardiovascular disease. $\mathrm{N}$ Engl J Med 1996;334:1150-5.

[38] The effect of vitamin $\mathrm{E}$ and beta carotene on the incidence of lung cancer and other cancers in male smokers. The Alpha-Tocopherol, Beta Carotene Cancer Prevention Study Group. N Engl J Med 1994;330:1029-35.

[39] Delcourt C, Cristol JP, Tessier F, Léger CL, Descomps B, Papoz L. Age-related macular degeneration and antioxidant status in the POLA study. POLA Study Group. Pathologies Oculaires Liées à l'Age. Arch Ophthalmol 1999;117:1384-90.

[40] Chong EW, Wong TY, Kreis AJ, Simpson JA, Guymer RH. Dietary antioxidants and primary prevention of age related macular degeneration: systematic review and meta-analysis. BMJ 2007;335:755.

[41] Klein BE, Knudtson MD, Lee KE, Reinke JO, Danforth LG, Wealti AM, et al. Supplements and age-related eye conditions the beaver dam eye study. Ophthalmology 2008;115:1203-8.

[42] Schreier P. Chemopreventive Compounds in the Diet. In: Augustin A, editor. Nutrition and the Eye. , Dev OphthalmolBasel: Karger; 2005. p. 1-58.

[43] Lonn E, Bosch J, Yusuf S, Sheridan P, Pogue J, Arnold JM, et al. Effects of long-term vitamin E supplementation on cardiovascular events and cancer: a randomized controlled trial. JAMA 2005;293:1338-47.

[44] Klein EA, Thompson Jr IM, Tangen CM, Crowley JJ, Lucia MS, Goodman PJ, et al. Vitamin E and the risk of prostate cancer: the Selenium and Vitamin E Cancer Prevention Trial (SELECT). JAMA 2011;306:1549-56.
[45] Mares-Perlman JA, Klein R, Klein BE, Greger JL, Brady WE, Palta $\mathrm{M}$, et al. Association of zinc and antioxidant nutrients with age-related maculopathy. Arch Ophthalmol 1996;114: 991-7.

[46] van Leeuwen R, Boekhoorn S, Vingerling JR, Witteman JC, Klaver CC, Hofman A, et al. Dietary intake of antioxidants and risk of age-related macular degeneration. JAMA 2005;294: 3101-7.

[47] VandenLangenberg GM, Mares-Perlman JA, Klein R, Klein BE, Brady WE, Palta M. Associations between antioxidant and zinc intake and the 5-year incidence of early age-related maculopathy in the Beaver Dam Eye Study. Am J Epidemiol 1998;148:204-14.

[48] Jacques PF, Halpner AD, Blumberg JB. Influence of combined antioxidant nutrient intakes on their plasma concentrations in an elderly population. Am J Clin Nutr 1995;62:1228-33.

[49] Parekh N, Chappell RJ, Millen AE, Albert DM, Mares JA. Association between vitamin $\mathrm{D}$ and age-related macular degeneration in the Third National Health and Nutrition Examination Survey, 1988 through 1994. Arch Ophthalmol 2007;125:661-9.

[50] Seddon JM, Reynolds R, Shah HR, Rosner B. Smoking, dietary betaine, methionine, and vitamin $\mathrm{D}$ in monozygotic twins with discordant macular degeneration: epigenetic implications. Ophthalmology 2011;118:1386-94.

[51] Millen AE, Voland R, Sondel SA, Parekh N, Horst RL, Wallace $\mathrm{RB}$, et al. Vitamin D status and early age-related macular degeneration in postmenopausal women. Arch Ophthalmol 2011;129:481-9.

[52] Kamburoglu G, Gumus K, Kadayifcilar S, Eldem B. Plasma homocysteine, vitamin B12 and folate levels in age-related macular degeneration. Graefes Arch Clin Exp Ophthalmol 2006;244:565-9.

[53] Rochtchina E, Wang JJ, Flood VM, Mitchell P. Elevated serum homocysteine, low serum vitamin B12, folate, and age-related macular degeneration: the Blue Mountains Eye Study. Am J Ophthalmol 2007;143:344-6.

[54] Christen WG, Glynn RJ, Chew EY, Albert CM, Manson JE. Folic acid, pyridoxine, and cyanocobalamin combination treatment and age-related macular degeneration in women: the Women's Antioxidant and Folic Acid Cardiovascular Study. Arch Intern Med 2009;169:335-41.

[55] Moriarty-Craige SE, Ha KN, Sternberg Jr P, Lynn M, Bressler S, Gensler G, et al. Effects of long-term zinc supplementation on plasma thiol metabolites and redox status in patients with age-related macular degeneration. Am J Ophthalmol 2007;143:206-11.

[56] Brantley Jr MA, Osborn MP, Sanders BJ, Rezaei KA, Lu P, Li C, et al. The short-term effects of antioxidant and zinc supplements on oxidative stress biomarker levels in plasma: a pilot investigation. Am J Ophthalmol 2012;153:1104-9.

[57] Newsome DA, Swartz M, Leone NC, Elston RC, Miller E. Oral zinc in macular degeneration. Arch Ophthalmol 1988;106: 192-8.

[58] Risk factors for neovascular age-related macular degeneration. The Eye Disease Case-Control Study Group. Arch Ophthalmol 1992;110:1701-8.

[59] Cho E, Stampfer MJ, Seddon JM, Hung S, Spiegelman D, Rimm EB, et al. Prospective study of zinc intake and the risk of agerelated macular degeneration. Ann Epidemiol 2001;11:328-36.

[60] Flood V, Smith W, Wang JJ, Manzi F, Webb K, Mitchell P. Dietary antioxidant intake and incidence of early age-related maculopathy: the Blue Mountains Eye Study. Ophthalmology 2002;109:2272-8.

[61] Kuzniarz M, Mitchell P, Flood VM, Wang JJ. Use of vitamin and zinc supplements and age-related maculopathy: the Blue Mountains Eye Study. Ophthalmic Epidemiol 2002;9:283-95.

[62] Age-Related Eye Disease Study Research Group. The effect of five-year zinc supplementation on serum zinc, serum 
cholesterol and hematocrit in persons randomly assigned to treatment group in the age-related eye disease study: AREDS Report No. 7. J Nutr 2002;132:697-702.

[63] Newsome DA. A randomized, prospective, placebo-controlled clinical trial of a novel zinc-monocysteine compound in age-related macular degeneration. Curr Eye Res 2008;33: 591-8.

[64] Beatty S, Chakravarthy U, Nolan JM, Muldrew KA, Woodside JV, Denny F, et al. Secondary outcomes in a clinical trial of carotenoids with coantioxidants versus placebo in early age-related macular degeneration. Ophthalmology 2012. http://dx.doi.org/10.1016/j.ophtha.2012.08.040 [Available online: http://www.aaojournal.org/article/S0161-6420(12)008500/fulltext (accessed on 07/01/2013)].

[65] Olin KL, Morse LS, Murphy C, Paul-Murphy J, Line S, Bellhorn RW, et al. Trace element status and free radical defense in elderly rhesus macaques (Macaca mulatta) with macular drusen. Proc Soc Exp Biol Med 1995;208:370-7.

[66] Selenium Flohé L. Selenoproteins and Vision. In: Augustin A, editor. Nutrition and the eye. , Dev OphthalmolBasel: Karger; 2005. p. 89-102.

[67] Tsang NC, Penfold PL, Snitch PJ, Billson F. Serum levels of antioxidants and age-related macular degeneration. Doc Ophthalmol 1992;81:387-400.

[68] Wong IY, Koo SC, Chan CW. Prevention of age-related macular degeneration. Int Ophthalmol 2011;31:73-82.

[69] Cho E, Hung S, Willett WC, Spiegelman D, Rimm EB, Seddon $\mathrm{JM}$, et al. Prospective study of dietary fat and the risk of age-related macular degeneration. Am J Clin Nutr 2001;73: 209-18.

[70] Smith W, Mitchell P, Leeder SR. Dietary fat and fish intake and age-related maculopathy. Arch Ophthalmol 2000;118:401-4.

[71] Heuberger RA, Mares-Perlman JA, Klein R, Klein BE, Millen AE, Palta M. Relationship of dietary fat to age-related maculopathy in the Third National Health and Nutrition Examination Survey. Arch Ophthalmol 2001;119:1833-8.

[72] Seddon JM, Rosner B, Sperduto RD, Yannuzzi L, Haller JA, Blair NP, et al. Dietary fat and risk for advanced age-related macular degeneration. Arch Ophthalmol 2001;119:1191-9.

[73] SanGiovanni JP, Chew EY, Clemons TE, Davis MD, Ferris III FL, Gensler GR, et al. The relationship of dietary lipid intake and age-related macular degeneration in a case-control study: AREDS Report No. 20. Arch Ophthalmol 2007;125:671-9.

[74] Chong EW, Robman LD, Simpson JA, Hodge AM, Aung KZ, Dolphin TK, et al. Fat consumption and its association with age-related macular degeneration. Arch Ophthalmol 2009;127:674-80.

[75] Sangiovanni JP, Agrón E, Meleth AD, Reed GF, Sperduto RD, Clemons TE, et al. \{omega\}-3 Long-chain polyunsaturated fatty acid intake and 12-y incidence of neovascular age-related macular degeneration and central geographic atrophy: AREDS report 30, a prospective cohort study from the Age-Related Eye Disease Study. Am J Clin Nutr 2009;90: 1601-7.

[76] Parekh N, Voland RP, Moeller SM, Blodi BA, Ritenbaugh C, Chappell RJ, et al. Association between dietary fat intake and age-related macular degeneration in the Carotenoids in Age-Related Eye Disease Study (CAREDS): an ancillary study of the Women's Health Initiative. Arch Ophthalmol 2009;127: 1483-93.

[77] Seddon JM, Cote J, Rosner B. Progression of age-related macular degeneration: association with dietary fat, transunsaturated fat, nuts, and fish intake. Arch Ophthalmol 2003;121:1728-37.

[78] Chiu CJ, Klein R, Milton RC, Gensler G, Taylor A. Does eating particular diets alter the risk of age-related macular degeneration in users of the age-related eye disease study supplements? Br J Ophthalmol 2009;93:1241-6.

[79] Hodge WG, Schachter HM, Barnes D, Pan Y, Lowcock EC, Zhang L, et al. Efficacy of omega-3 fatty acids in preventing age-related macular degeneration: a systematic review. Ophthalmology 2006;113:1165-72.

[80] Chong EW, Kreis AJ, Wong TY, Simpson JA, Guymer RH. Dietary omega-3 fatty acid and fish intake in the primary prevention of age-related macular degeneration: a systematic review and meta-analysis. Arch Ophthalmol 2008;126:826-33.

[81] Hodge WG, Barnes D, Schachter HM, Pan YI, Lowcock EC, Zhang L, et al. Evidence for the effect of omega-3 fatty acids on progression of age-related macular degeneration: a systematic review. Retina 2007;27:216-21.

[82] Lawrenson JG, Evans JR. Omega 3 fatty acids for preventing or slowing the progression of age-related macular degeneration. Cochrane Database Syst Rev 2012;14(11):CD010015. http://dx.doi.org/10.1002/14651858. [http://onlinelibrary. wiley.com/doi/10.1002/14651858.CD010015.pub2/pdf (accessed on 09/01/2013)].

[83] Mares-Perlman JA, Brady WE, Klein R, VandenLangenberg GM, Klein BE, Palta M. Dietary fat and age-related maculopathy. Arch Ophthalmol 1995;113:743-8.

[84] Jenkins DJ, Kendall CW, McKeown-Eyssen G, Josse RG, Silverberg J, Booth GL, et al. Effect of a low-glycemic index or a high-cereal fiber diet on type 2 diabetes: a randomized trial. JAMA 2008;300:2742-53.

[85] Chiu CJ, Milton RC, Gensler G, Taylor A. Association between dietary glycemic index and age-related macular degeneration in nondiabetic participants in the Age-Related Eye Disease Study. Am J Clin Nutr 2007;86:180-8.

[86] Chiu CJ, Hubbard LD, Armstrong J, Rogers G, Jacques PF, Chylack Jr LT, et al. Dietary glycemic index and carbohydrate in relation to early age-related macular degeneration. Am J Clin Nutr 2006;83:880-6.

[87] Chiu CJ, Taylor A. Dietary hyperglycemia, glycemic index and metabolic retinal diseases. Prog Retin Eye Res 2011;30: 18-53.

[88] Uchiki T, Weikel KA, Jiao W, Shang F, Caceres A, Pawlak D, et al. Glycation-altered proteolysis as a pathobiologic mechanism that links dietary glycemic index, aging, and agerelated disease (in nondiabetics). Aging Cell 2012;11:1-13.

[89] Mallal S, Phillips E, Carosi G, Molina JM, Workman C, Tomazic J, et al. HLA-B*5701 screening for hypersensitivity to abacavir. N Engl J Med 2008;358:568-79.

[90] Stocchi L, Cascella R, Zampatti S, Pirazzoli A, Novelli G, Giardina E. The pharmacogenomic HLA biomarker associated to adverse abacavir reactions: comparative analysis of different genotyping methods. Curr Genomics 2012;13:314-20.

[91] Berger CT, Kim AY. IL28B polymorphisms as a pretreatment predictor of response to HCV treatment. Infect Dis Clin North Am 2012;26:863-77.

[92] Muir AJ. IL28B in the era of direct-acting antivirals for hepatitis C. J Clin Gastroenterol 2013;47:222-7.

[93] Mares JA, Voland RP, Sondel SA, Millen AE, Larowe T, Moeller $\mathrm{SM}$, et al. Healthy lifestyles related to subsequent prevalence of age-related macular degeneration. Arch Ophthalmol 2011;129:470-80. 